\title{
Bearing capacity analysis of helical pile foundation on peat
}

\author{
Ferry Fatnanta ${ }^{1}$ and Andarsin Ongko ${ }^{1, *}$ \\ ${ }^{1}$ Civil Engineering Department, University of Riau, Indonesia
}

\begin{abstract}
Peat is a kind of soil with a very low bearing capacity and high compressibility. Generally, a building construction on peat is done by using a wooden pile foundation. However, the length of the wooden piles is sometimes limited and causes the friction strength between the soil and wooden piles to became suboptimal. In order to enhance the bearing capacity of the foundation, the cross-sectional area of the foundation needs to be enlarged. One of the solutions for this problem is through helical piles. There are two methods to determine the helical pile's bearing capacity, i.e. individual bearing and cylindrical shear methods. In this paper, bearing capacity prediction was discussed. A foundation load test was thoroughly done by a constant rate of penetration. This test consisted of compression and tension tests. The result was analyzed by individual bearing and cylindrical shear methods and next compared to each other. The result of the analysis has shown that the individual bearing method was more suitable in predicting helical piles' bearing capacity since it produced the lowest error rate, with a magnitude of $21,31 \%$.
\end{abstract}

Keywords: helical pile foundation, peat, individual bearing and cylindrical shear method

\section{Introduction}

The texture of peat is dominated by voids and large amounts of water. This condition causes great compressibility. Due to the high-water content, peat can be categorized as very soft soil with a very low bearing capacity.

In order to overcome these problems, lightweight building constructions on peat are generally done either by wooden piles or conventional piles. To keep it economical, pile foundations were embedded without reaching the bed rock layer. Hence, the compressive bearing capacity of the foundations rely on the friction between the soil layer and foundations, which make the foundation's bearing capacity not optimal. One of the ways to enhance the foundation's bearing capacity is by enlarging the cross-sectional area of foundations, using helical plate installation on the edge of pile foundations.

There is still a lack of information and research about the utilization of helical pile foundations on peat. There are two methods to calculate the bearing capacity of helical pile

\footnotetext{
*Corresponding author: andarsin.ongko@student.unri.ac.id
} 
foundations, i.e. the individual bearing method and cylindrical shear method. These methods have given a different bearing capacity value. Therefore, in this study, these two methods will be reviewed to determine which method is more suitable to be applied in peat case studies.

\section{Mechanisms of helical pile foundation`s bearing capacity}

According to [1], there are two methods to determine a helical pile foundation's bearing capacity, i.e. individual bearing and cylindrical shear, as in figure 1. In case of a major space between helical plates, each helical plate is able to act individually as an end bearing holder. Therefore, the bearing capacity of the helical pile foundation is the sum total of the helical plates' individual capacity. This is called individual bearing, as shown in figure 1a.

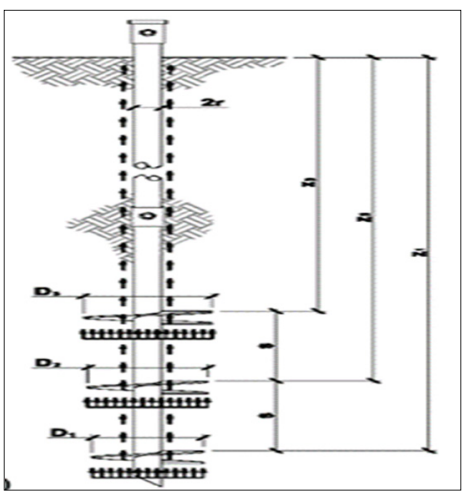

(a)

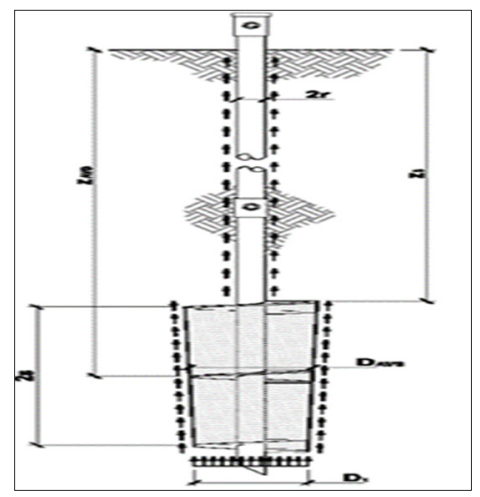

(b)

Fig. 1. Individual bearing and cylindrical shear method.

However, in case of a minor distance between helical plates, the mechanisms will be different. All helical plates will be working together. So, the bearing capacity of the helical pile foundation is a combination of the bearing capacity on the base of the helical plate and the friction force along the soil between helical plates. This is called cylindrical shear, as displayed in figure $1 \mathrm{~b}$.

\subsection{Individual bearing method}

The individual bearing method assumes that each helical plate becomes a bearer/footing. Therefore, a uniform stress distribution occurs below each helical plate. Limit bearing capacity $(\mathrm{Pu})$ in this method can be obtained from the equation below:

$$
P_{u}=\sum_{n} q_{u l t} A_{n}+\alpha H(\pi d)
$$

Where $\mathrm{qult}_{\mathrm{u}}=$ limit bearing capacity below helical plate; $\mathrm{A}_{\mathrm{n}}=\mathrm{n}$-area of helical plate; $\alpha=$ adhesion between soil and pile surface. The value of $\alpha$ was taken from the friction angle between the foundation material and soil layers; $\mathrm{H}=$ length of the pile foundation (from uppermost helical plate until soil surface); $d=$ diameter around the shaft (diameter of pile foundation without helical plate).

In some cases of fine grained soil, $\phi=0$, then $\mathrm{N}^{\prime}{ }_{\mathrm{c}} \approx 10$. For deep foundations, [2] has found that the $\mathrm{qult}_{\mathrm{u}}$ for fine grained soil is:

$$
q_{u l t}=9 c_{u}
$$




\subsection{Cylindrical shear method}

In the cylindrical shear method, value of Pu can be defined as:

$$
P_{u}=q_{u l t} A_{1}+T(n-1) s \pi D_{\text {avg }}+\alpha H(\pi D)
$$

Where, $A_{1}=$ area of lowermost helical plate, $\mathrm{T}=$ soil's shear strength, $\mathrm{H}=$ length of the foundation shaft (from uppermost helical plate until soil surface), $d=$ diameter of the pile foundation, $D_{\text {avg }}=$ average diameter of the helical plate, $(n-1) s=$ soil space between helical plates, $\mathrm{n}=$ number of helical plates, $\mathrm{s}=$ space between helical plates. In case of fine grained soil, T (soil's shear strength) is taken as undrained shear $\left(\mathrm{c}_{\mathrm{u}}\right)$.

\section{Research methodology}

\subsection{Research location and materials}

Peat materials were taken from Rimbo Panjang, Kampar District. Physical and mechanical property tests of the peat were done in the Soil Mechanics Laboratory of the Department of Civil Engineering, University of Riau. The scheme of the test can be observed in figure 2.

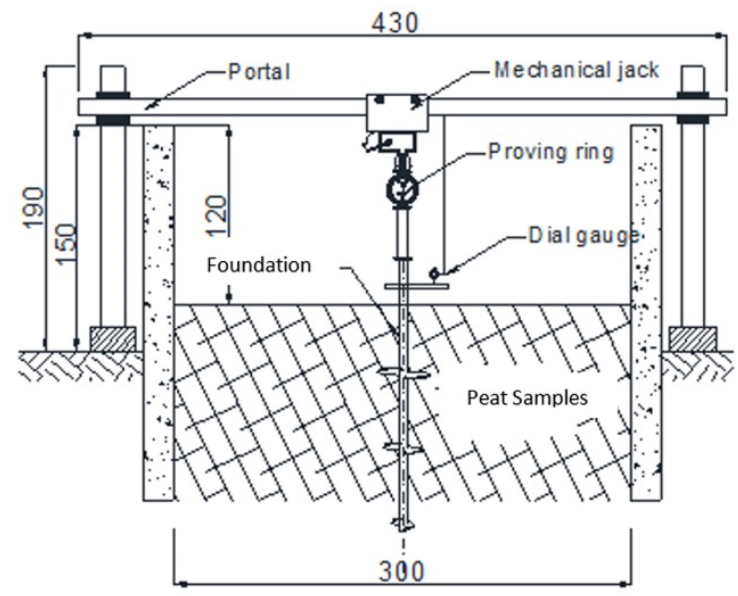

Fig. 2. Scheme of testing model.

The peat was kept natural, corresponding to field conditions. No compaction was applied during the sample preparation. The properties test consisted of water content, unit weight, specific gravity, fiber and ash content. The results can be observed in Table 1.

Table 1. Peat's properties.

\begin{tabular}{|l|l|l|}
\hline Test(s) & Unit & Value \\
\hline Water content & $\%$ & 242,39 \\
\hline Wet density & $\mathrm{gr} / \mathrm{cm}^{3}$ & 0,82 \\
\hline Dry density & $\mathrm{gr} / \mathrm{cm}^{3}$ & 0,24 \\
\hline Specific gravity & - & 1,58 \\
\hline Ash content & $\%$ & 32,80 \\
\hline Fiber content & $\%$ & 9,58 \\
\hline Void ratio & - & 5,61 \\
\hline
\end{tabular}

According to Table 1 and [3], the peat samples could be categorized as high ash peat. Whereas according to [4], the peats are part of sapric peat based on their fiber content. 


\subsection{Shape of helical pile`s foundation and nomenclature naming}

The length of the helical pile's foundation reached $2 \mathrm{~m}$ with a diameter of $6 \mathrm{~cm}$. The helical plate's diameter was $15 \mathrm{~cm}$ (Small Plate), $25 \mathrm{~cm}$ (Medium Plate), and $35 \mathrm{~cm}$ (Large Plate).

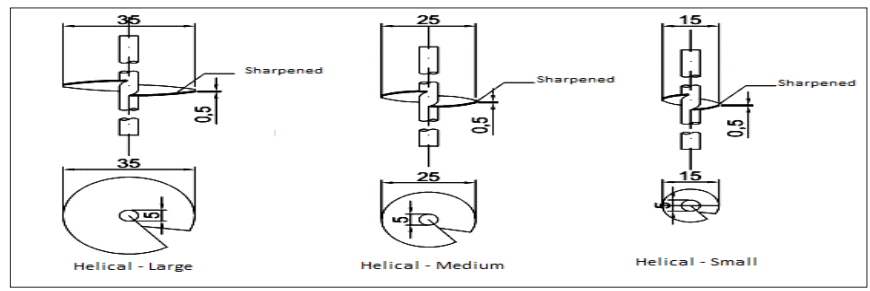

Fig. 3. Diameter of helical plates.

For example, if a helical pile foundation is mentioned as LMS 50, this means the foundation was equipped with 3 plates with the top section as L (Large), the middle section as $\mathrm{M}$ (Medium), and the bottom section as S (Small), with a gap between each plate of 50 $\mathrm{cm}$, as it can be observed in figure 4 .

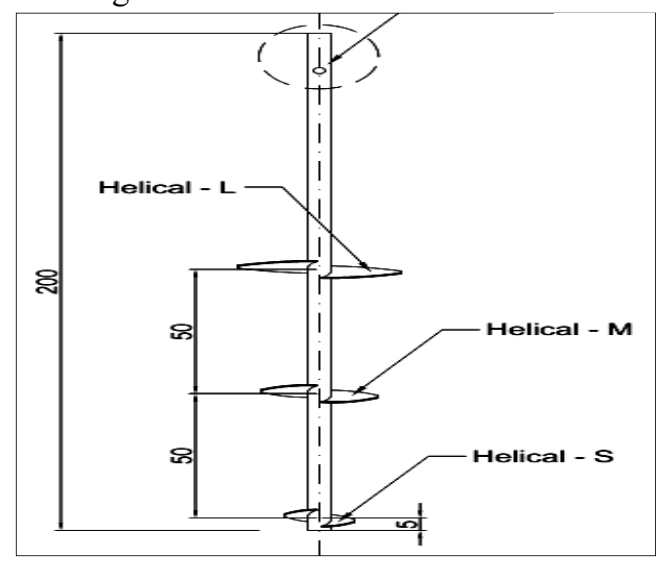

Fig. 4. Helical pile foundation type LMS 50.

A list of helical pile foundation models can be observed in table 2.

Table 2. Nomenclature naming of helical pile foundation.

\begin{tabular}{|c|c|c|c|}
\hline No & Name & No & Name \\
\hline 1 & M & 9 & LMS50 \\
\hline 2 & L & 10 & LL20 \\
\hline 3 & LM20 & 11 & LL30 \\
\hline 4 & LM30 & 12 & LL50 \\
\hline 5 & LM50 & 13 & LLL20 \\
\hline 6 & LMS20 & 14 & LLL30 \\
\hline 7 & LMS30 & 15 & LLL50 \\
\hline 8 & LMS50 & & \\
\hline
\end{tabular}

\subsection{Test procedures and determination of limit bearing capacity of helical pile foundation}

Foundations were embedded until they reached a depth of $1,5 \mathrm{~m}$. Before the test was initiated, the peat was tested by a field vane shear test for depths of 50,100 , and $150 \mathrm{~cm}$. The compress/ 
tensile test was used a constant rate of penetration method. Some equipment were used, i.e. mechanical jack, proving ring, and dial gauge [5]. The rate of penetration was $2 \mathrm{~mm} / \mathrm{minute}$. The load was recorded for each $1 \mathrm{~mm}$ settlement. The maximum settlement of the foundation was $45 \mathrm{~mm}$. It corresponded to the capacity of the dial gauge.

The limit bearing capacity of the foundation was interpreted by the Terzaghi and Peck method. The limit bearing capacity of the foundation satisfied the settlement of $25 \mathrm{~mm}$, as shown in figure 5 . According to figure 5, the limit bearing capacity of the foundation was 8,3 $\mathrm{kN}$. This method is much easier and can be interpreted equally for all load versus settlement data.

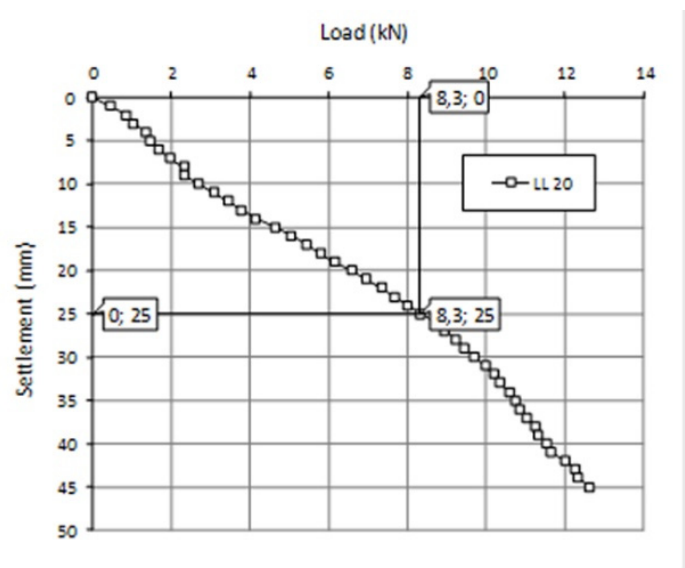

Fig. 5. Interpretation of limit bearing capacity on helical pile foundation.

\section{Results and discussion}

The shear strength test on peat was done for each type of foundation through a Vane Shear Test [6]. According to figure 6, it can be observed that the shear strength of the peat was between 3,00 to $5,25 \mathrm{kPa}$ when the samples were loaded with compressive action. This also shows that the peat was homogeneous.

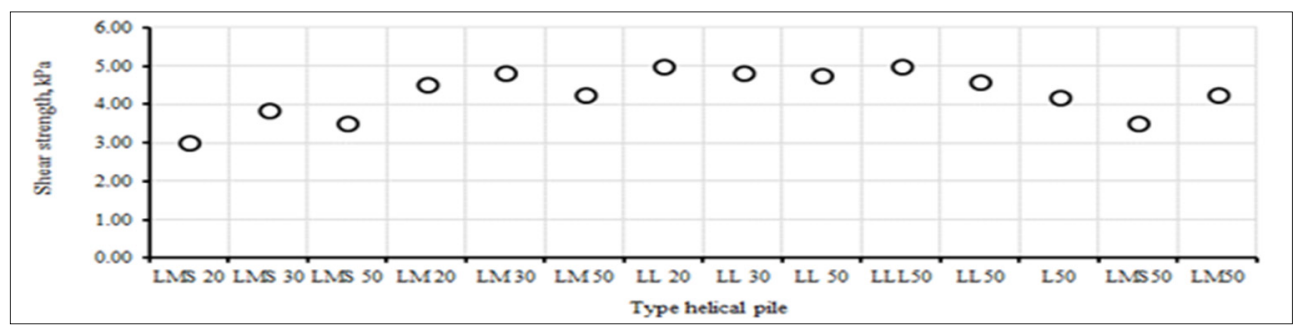

Fig. 6. Shear strength of peat on compressive test.

Whereas figure 7 shows the shear strength of the peat was between 4,80 to 7,50 $\mathrm{kPa}$ when the samples were loaded with tensile action. 


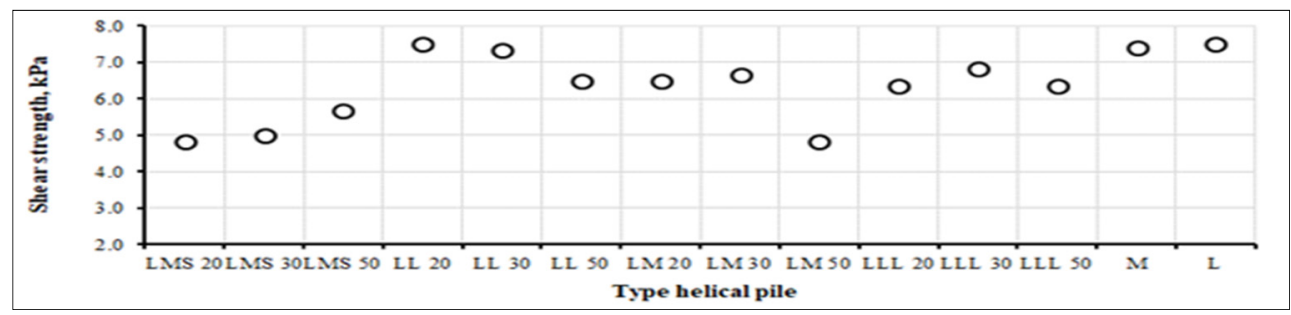

Fig. 7. Shear strength of peat on tensile test.

\subsection{Comparisons between helical pile foundation, wooden pile, and non-helical pile foundation}

In figure 8 , it is shown that the installation of the helical plate significantly enhanced the bearing capacity of the foundation. Pile foundation LMS 50 had a greater bearing capacity compared to wooden pile and non-helical pile foundations, but this type had the lowest bearing capacity between the helical piles. This was followed by the wooden pile, with the coarser surface. Lastly, the non-helical pile foundation gained the lowest bearing capacity.

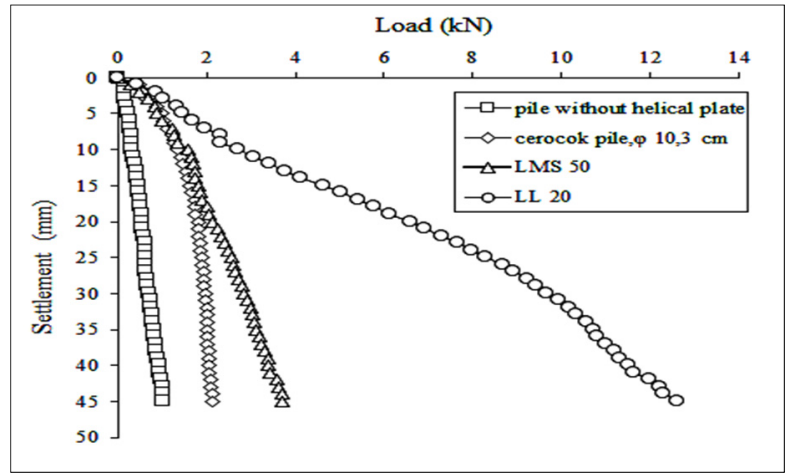

Fig. 8. Comparisons of load versus settlement for all materials.

\subsection{Prediction and compressive bearing capacity test}

The analysis of the helical pile foundation's bearing capacity was thoroughly done through 2 methods, i.e. the individual bearing and cylindrical shear method. The result of test can be observed in figure 9.
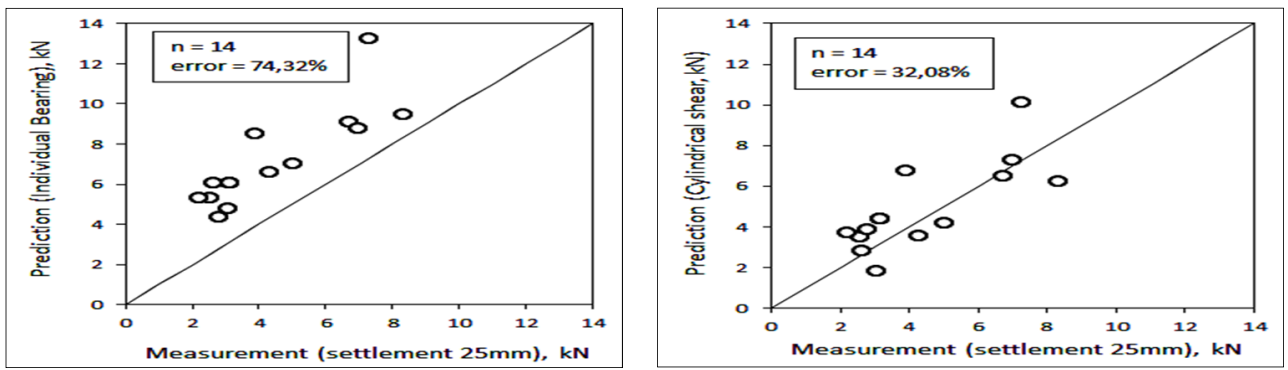

Fig. 9. Comparisons of helical pile foundation`s compressive bearing capacity. 
According to figure 9, the error rate for the individual bearing and cylindrical shear were respectively $74,32 \%$ and $32,08 \%$. [7] has suggested to reduce the peat's soil strength with a factor of 0,5 . Based on that consideration, the reduction was done in order to obtain lower error rates. Figure 10 displays that the individual bearing method has given the closest result to the test result, with an error rate of $19,72 \%$.
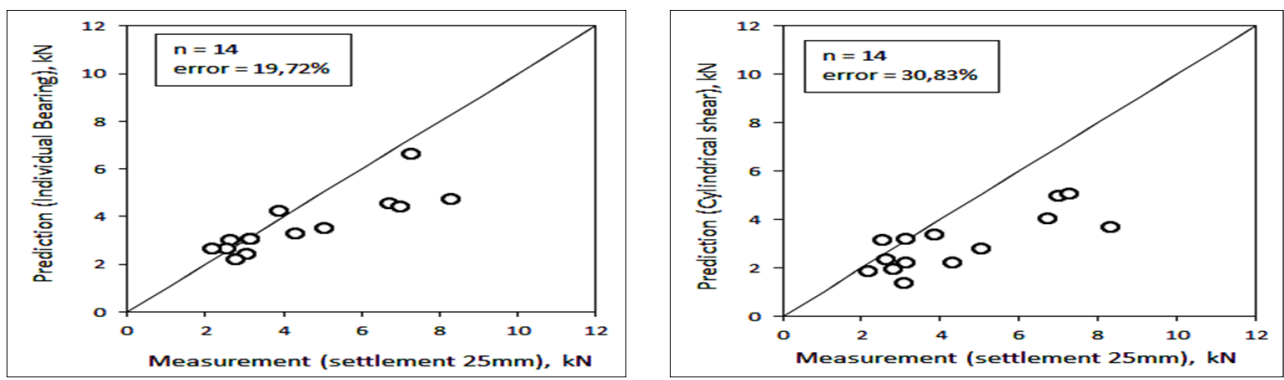

Fig. 10. Comparisons of helical pile foundation's compressive bearing capacity with reduction factor case.

\subsection{Prediction and tensile bearing capacity test}

A vertical tensile test was done on the helical pile. Similarly to the compressive test, in this prediction, there are 2 methods to forecast the helical foundation's bearing capacity. According to figure 11, without any strength reduction, the individual bearing method has produced an error rate of $165,05 \%$; as for the cylindrical shear method, this reached $77,69 \%$.
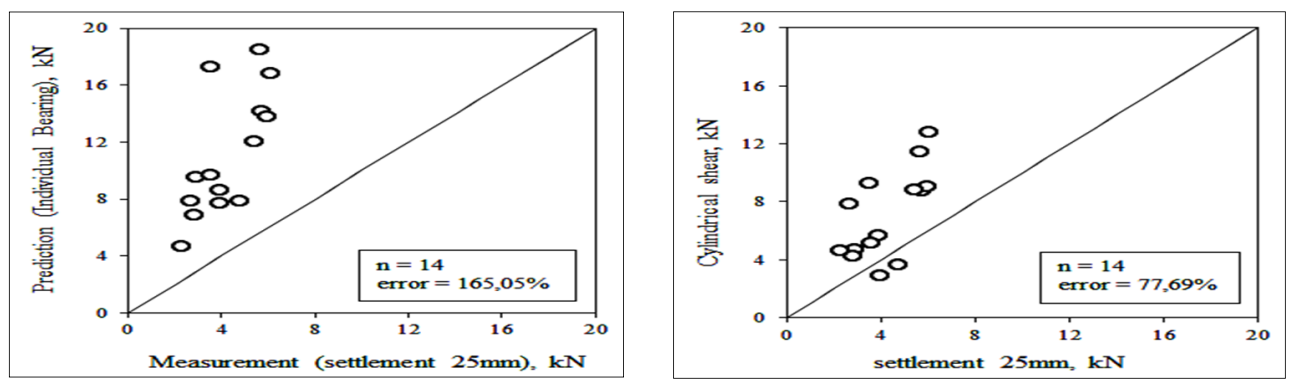

Fig. 11. Comparisons of helical pile foundation`s tensile bearing capacity.

The same reduction case applied on the tensile test case. However, the reduction value was only 0,37 . In figure 12 , it is shown that the error rate of individual bearing in predicting the tensile bearing capacity has reached $21,32 \%$. The cylindrical method reached an error rate of $28,45 \%$.
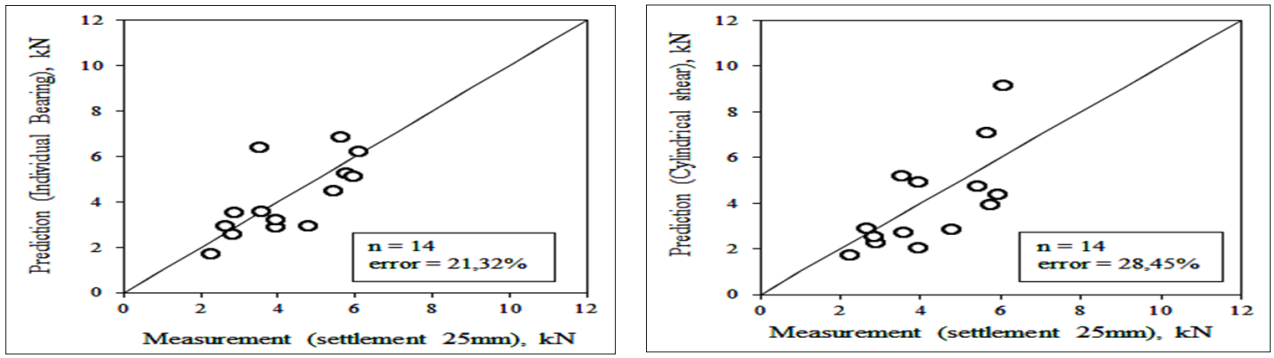

Fig. 12 Comparisons of helical pile foundation`s tensile bearing capacity with reduction factor case. 


\section{Conclusions}

From the discussion results, it could be concluded that:

1. The helical pile foundation has shown a greater bearing capacity on peat, compared to wooden piles and non-helical pile foundations.

2. Corresponding to the error rate value, the individual bearing method was more suitable for the prediction of compressive bearing capacity on helical pile foundations. The same applies for the prediction of tensile bearing capacity. They produced the lowest error rate value. In this paper, the lowest error rate could be reached by using a reduction factor of 0,37 .

\section{References}

1. H. Perko, Helical Piles (A Pratical Guide to Design and Installation), (John Wiley \& Sons, Inc, 2009).

2. A.W. Skempton, The Bear. Cap. of Clays, Building Research Congress, 180-189, (1951).

3. U.S.A: Annual Book of ASTM Standards sec 4, ASTM D2974-00. Standard Test Method for Moisture, Ash, and Organic Matter of Peat and Other Organic Soils. (2000).

4. U.S.A: Annual Book of ASTM Standards sec 4, ASTM D1997-91. Standard Test Method for Laboratory Determination of The Fiber Content of Peat Samples by Dry Mass. (2001).

5. U.S.A: Annual Book of ASTM Standards sec 4, ASTM D1143-81. Standard Test Method for Piles Under Static Axial Compressive Load. (1994).

6. U.S.A: Annual Book of ASTM Standards sec 4, ASTM D2573-01. Standard Test Method for Field Vane Shear Test in Cohesive Soils. (2001).

7. A. Golebiewska, V. Test. in Peat, Proceeding 7th Danube ECSMFE, 1, 113-117, (1983). 